\title{
Different Levels of EGF, VEGF, IL-6, MCP-1, MCP-3, IP-10, Eotaxin and MIP-1 $\alpha$ in Adipose Derived Stem Cells (ADSCs) Secretome in Androgenetic Alopecia
}

Katarina Andjelkov ( $\nabla$ kandjelkov@belprimeclinic.com )

University of Belgrade, Faculty of Medicine https://orcid.org/0000-0002-3341-8660

llya Eremin

Laboratory for cell biology and developmental pathology

Aleksandra Korac

University of Belgrade, Faculty of Biology

\section{Research}

Keywords: hair follicle, androgenetic alopecia, adipose derived stem cells

Posted Date: January 4th, 2021

DOI: https://doi.org/10.21203/rs.3.rs-137172/v1

License: (c) (i) This work is licensed under a Creative Commons Attribution 4.0 International License.

Read Full License 


\section{Abstract}

\section{BACKGROUND}

Hair follicles and underlying adipose tissue show highly coordinated interaction through exchange of different signaling molecules. We conducted a study to investigate the quantitative and qualitative secretome profiling of Adipose Derived Stem Cells (ADSCs) from different zones of hair growth in patients with Androgenetic Alopecia (AGA).

\section{METHODS}

We included 6 male patients, candidates for follicular unit extraction hair transplantation, all in early stage of AGA. $1 \mathrm{~mm}$ punch samples of adipose tissue located beneath hair follicles of 3 scalp areas (alopecia, border-line and normal hair growth) and 1 periumbilical sample were analyzed.

Samples were enzymatically digested, centrifuged, washed, and cell pellets were ceded and maintained in culture medium until reached monolayer. Conditioned media samples were thawed and analyzed with 41 plex kit. Results were registered by Magpix device (Luminex platform) and calculated with xPonent software. We analyzed the levels of 35 signaling proteins.

\section{RESULTS}

The level of Inteleukin-6 (IL-6) were significantly higher in the alopecia zone in comparison to the periumbilical and occipital, alongside with the levels of Vascular Endothelial Growth Factor (VEGF), Endothelial Growth Factor (EGF) and Eotaxin. The similar trend was found for Monocyte Chemotactic Protein-3 (MCP-3), Interferon gamma-inducible Protein-10 (IP-10) and Macrophage Inflammatory Protein - 1 alpha (MIP-1 $\alpha$ ), while, on the other side, Monocyte Chemoattractant Protein-1 (MCP-1) level was the lowest in alopecia comparing to other zones. All other examined proteins did not shown changes in their expression level.

\section{CONCLUSION}

Hence, knowledge of differences in these signaling molecules expression will be essential for both, achieving therapeutic goals for hair loss conditions and shading more lights on the AGA etiology.

\section{Introduction}

The entire mature anagen hair follicle (HF) is embed in dermal white adipose tissue (dWAT) and their long and short-range interactions involve the exchange of a vast variety of signaling molecules which are released by different cells found within the adipose tissue. [1, 2]

That interaction was a scientific background of the adipose derived regenerative cell-based therapy for androgenetic alopecia (AGA), introduced with the potential to revolutionize the treatment for androgenetic 
alopecia. Unfortunately, conducted clinical trials have made only modest improvements in restoring hair growth. This limitation is due, at least in part, to the death of transplanted cells within a few hours after transplantation. The cause of rapid death was due to mutual effects of different mechanical, cellular and host factors. [3]

Mesenchymal stem cells (MSCs), widely present in dWAT exert many of their effects via paracrine signaling. $[4,5]$ Therefore, there are attempts of replacing the cells with their secretome for many, including this therapeutic application. [6-8]

According to some recent reviews, several hurdles need to be addressed in the clinical translation of this potentially new therapeutical tool. One of the most important steps is to classify the cell secretome, bioactive signals produced by mesenchymal cells that can be secreted as soluble molecules or packed into more complex structures named extracellular vesicles. The next should be to determine the method of administration and harmonize guidelines and protocols. So far, clinical translation has been limited to local applications, such as one for hair loss. [7] The application of MSC-secretome in androgenetic alopecia could be more promising tool then conventional stromal-vascular fraction (SVF) cell therapy since they convey a definite set of signals with more limited and predictable effects. The production of these biological "nanoparticles" is less complex compared to living cells, cheaper and circumventing a major barrier in the diffusion of these innovative treatments. [3] Administration of specific secretome in patients with AGA may represent an alternative and innovative strategy for cell-free cell therapy. Thus, there is urgent need to understand the composition of ADSCs secretome in order to defining an optimal "cocktail" to preconditioning hair follicles for a given therapeutic application.

Hence, we aimed this study to establish the composition of ADSCs secretome from 3 scalp hair growth areas (alopecia, border-line and normal hair growth) and 1 periumbilical sample in patients with AGA.

Our hypothesis was that adipocyte-derived signaling molecules may allow distinct interactions between HFs and DWAT and therefore, may vary in their composition and their quantity in different areas of hair growth in patients with androgenetic alopecia. Furthermore, we hypothesized that they may play an important role in the onset of androgenetic alopecia.

\section{Methods}

The inclusion criteria for the study were: male patients, age between 18 and 60 years old, candidates for Follicular Unit Extraction (FUE) technique for hair transplantation. All were clinically diagnosed with AGA type III (Norwood-Hamilton scale) by a clinical dermatologist, with no other comorbidities and under no other (either systemic or topical) treatment for AGA in passed 1 year.

The exclusion criteria were: other types of alopecia, more advanced stages of AGA (more than stage III according to the Norwood-Hamilton scale), presence of a skin disease, infections or other comorbidities and under some other therapeutic treatment for AGA in past 1 year. 
A specially developed patient's card was created including information about age, the anamnesis and features of AGA, allergies, social habits, early conducted treatments, etc.

Human scalp tissue biopsies were obtained with patient's signed consent during the FUE hair transplantation. The protocol used in the study was approved by the Colic Hospital Ethics Committee and all participants provided written informed consent. The study was conducted in accordance with the Declaration of Helsinki.

Biopsies were performed with the use of 1-mm disposable biopsy punch under local anesthesia ( $1 \%$ Lidocaine with 1:200 000 epinephrine solution), one from each of following locations (Fig. 1):

1. Occipital zone (donor area for hair transplants) - normal hair growth area

2. Border-line scalp zone - "battle field area" - area where there is still a hair growth, but the hair is thinner and scarce

3. Alopecia zone - "lost battle field" - area with no hair growth, recipient area for hair transplants

4. Periumbilical zone

All samples were collected into the sterile tubes contained transport medium, Dulbecco's Modified Eagle Medium (DMEM F12) supplemented with $5 x$ anti-anti solution (all from Gibco, USA) and were delivered to the laboratory for analysis within two hours from collection.

\section{Obtaining cell cultures and conditioned medium samples}

In the laboratory all samples were enzymatically digested with collagenases mix - collagenase I and collagenase II (all from Gibco, USA) in 1:1 ratio for $10 \mathrm{~min}$ on the rotated shaker chamber $120 \mathrm{rpm}$, at $37^{\circ} \mathrm{C}$.

After digestion collagenase activity was inhibit by adding a three times volume of Dulbecco's PhosphateBuffered Saline (DPBS) without $\mathrm{Ca}^{2+}$ and $\mathrm{Mg}^{2+}$ (Gibco, USA). Tubes with digested samples were centrifuged for $10 \mathrm{~min}, 300 \mathrm{~g}$, at $25^{\circ} \mathrm{C}$. Cell pellets were resuspended in DPBS and rinsed by centrifugation.

Finally, cell pellets were ceded with DMEM $\backslash$ F12, culture medium supplemented with $10 \%$ of Fetal Bovine Serum (FBS) (HyClone, GE, USA) for 10 days in $5 \% \mathrm{CO}_{2}$ at $37^{\circ} \mathrm{C}$. Culture medium was changed from day 10 every 3-4 days for getting monolayer culture.

After cell cultures reached monolayer, culture medium was changed for serum-free for 3 days. After this time culture medium was collected and stored at $-70{ }^{\circ} \mathrm{C}$ in aliquots. Cells were detached with Gibco TrypLE Express enzyme solution and cryopreserved for the future testing.

\section{Multiplex analysis}


Conditioned media samples were thawed and analyzed in doubles immediately with 41 plex kit (xMap technology, Millipore, USA) according to manufacturer protocol. Results were registered by Magpix device (Luminex platform) and calculated with xPonent software (Luminex, Austin, TX).

\section{Statistical analyzes}

Collected data were analyzed by using statistical software PASW® Statistic v. 25 (Chicago, Illinois, USA). Data were presented as medians and 25th - 75th percentile values in parenthesis, because all parameters deviated from normal distribution. For signaling molecules concentration in 4 different adipose tissue samples comparison, Friedman's non-parametric test for several related samples was used, with subsequent Wilcoxon's paired test for group by group comparison. Receiver operating characteristic curve (ROC) analysis was used in order to test predictive capability of analyzed biomarkers regarding adipose tissue area. Binary logistic regression analysis was used for predictive probabilities calculation derived from two-parameters model and these new variables was used for additional ROC analysis. Levels of examined signaling molecules in different subgroups were presented graphically by box plot graphs.

\section{Results}

From October 2018 until February 2019 we have analyzed samples of 6 patients.

Alopecia patients were middle-aged, lean male subjects in stage III, according to the Hamilton scale for male Androgenetic Alopecia. They were mostly non-smokers (only one patient is a current smoker). They all were healthy patients, with no previous treatment for AGA. All samples were collected during their FUE hair transplant. (Table 1.)

We analyzed 35 of secreted molecules belonging to 5 different groups of signaling molecules: proinflammatory, anti-inflammatory, chemokines, stimulators of cell growth and differentiation and regulatory one. All of them were present in all of 4 examined zones.

From the proinflammatory group of secreted molecules, Interleukin-6 (IL-6) was the only with significantly lower level in periumbilical zone of adipose tissue compared to alopecia zone. Among alopecia zones, level of IL-6 were the lowest in occipital zone, following the battle field zone and the highest in alopecia zone, but the difference between the three scalp adipose tissue areas was not statistically significant probably due to a large inter-individual variability. However, a trend towards higher proinflammatory status was noted in the alopecia affected area.

We have found a similar situation for Vascular Endothelial Growth Factor (VEGF) - its level was significantly lower in periumbilical zone adipose tissue compared to alopecia zone. Comparing the three scalp zones we also noticed that the lowest VEGF level was in the occipital zone, then in the battle field zone and the highest levels were in the alopecia zone, but again, without statistical significance.

Endothelial Growth Factor (EGF) level was the lowest in the periumbilical tissue zone and significantly lower in comparison to all other analyzed zones. (Fig. 2.) 
The eotaxin level was the lowest in periumbilical adipose tissue under the hair follicle and it was significantly decreased compared to the other three tissues' samples. The battle field zone showed the lowest level of eotaxin, alopecia zone the highest, but this difference didn't reach a significant difference. The level of Monocyte Chemoattractant Protein-1 (MCP-1) was the lowest in the alopecia zone compared to the other two scalp zones and to the periumbilical tissue samples as well. The Monocyte Chemotactic Protein-3 (MCP-3) level, if detectable, was very low in the periumbilical zone, suggesting potential lack of function in this area. The significantly higher MCP-3 level was observed in the alopecia zone compared to occipital. Borderline zone had a similar level of this protein as the alopecia zone, suggesting an involvement of eosinophils leukocytes' subspecies in inflammatory processes associated with alopecia and alopecia harbinger. (Fig. 3.)

The levels of Interferon gamma-inducible Protein (IP-10) and Macrophage Inflammatory Protein - 1 alpha (MIP-1a) showed a similar pattern of difference between adipose tissue samples from the different scalp areas. Namely, the level of both signaling molecules were significantly higher in alopecia area compared to borderline area.

All molecules which have shown significant difference, regarding tissue alopecia status and specific localization, were subjected to correlation analysis. This analysis showed significant, positive and strong correlation between almost all analyzed proteins. (Table 2.)

In order to test diagnostic accuracy of the analyzed signaling molecules towards distinct alopecia zone's origin with central alopecia zone as a reference (state variable) and occipital zone as a comparative zone, we have performed receiver operating characteristics curve (ROC) analysis and tested all 8 proteins which in primary analysis had given significant difference between subgroups. Initial analysis didn't show any of the protein had significant diagnostic accuracy in alopecia status diagnosis. After the binary logistic regression analysis implementation and calculation of predicted probabilities of different proteins combination we have found that VEGF and MCP-1 combination (as merged model of parameters) had significant diagnostic accuracy. Area under the curve with 95th $\mathrm{Cl}$ and significance of the curve derived from predicted probabilities of two protein combinations is presented in Table 3. Regarding VEGF and MCP-1 concentration, having in mind its values in alopecia zone tissue vs. occipital zone tissue (Table 3.) we suppose that combination of VEGF values above $573 \mathrm{pg} / \mathrm{mL}$ ( $>75$ th percentile in occipital tissue) and MCP-1 value below $10670 \mathrm{pg} / \mathrm{mL}$ (<25th percentile for occipital tissue) show significant tissue activity caused by alopecia inflammation.

\section{Discussion}

Among many studies already published regarding the leading cause and etiology of AGA, two lines of evidence have emerged and point out androgen metabolism and inflammation. However, as treatment of AGA with either androgen metabolism modulators or antihypertensive agents does not exceed $30 \%$ of success rate, other etiology pathways may be involved. [9] Several studies also showed that inflammation and fibroplasia of the hair follicle's dermal sheath can lead to downsizing and involution of 
the pilosebaceous unit in AGA. [9-13] One of the possible cofactor of this initial pro-inflammatory stress could be a presence of several microbial inhabitants in the scalp and/or their products. [14] Balding dermal papilla (DP) cells also produce higher levels of inflammatory cytokines, such as IL-6. IL-6 not only inhibits anagen entry, but also disrupts normal anagen progression. Our results of a positive trend towards higher levels of proinflammatory IL- 6 along with significantly higher levels of chemokines (eotaxin, MCP-3, IP-10, MIP-1 $\alpha$ ) in alopecia affected area clearly support this idea.

The newly identified C-C chemokine, MCP-3, is chemotactic for and activates a variety of inflammatory cells. [15] MCP-3 has 71\% amino acid identity to MCP-1, and both activate monocytes, T cells, and basophils. However, MCP-3, unlike MCP-1, also activates eosinophils. On the other hand, MIP1a and Rantes which are about 30\% identical to MCP-3, both also activate eosinophils, in addition to monocytes, T lymphocytes and basophils. [15-18] MCP3 has been proposed to interact with the receptors for MCP-1, MIP-1a, and Rantes. [15] We were able to measure all these chemokines. As previously said, the levels of MCP-3 were almost lacking in the periumbilical zone and in the occipital zone (zones of normal hair growth), while they were significantly elevated in the alopecia zone. Decreased levels of MCP-3 in the occipital zone may reflect calm inflammatory state, irrespective of the presence of inflammatory cells. Surprisingly, the situation with MCP-1 was the opposite; its levels were the lowest in the alopecia zone and also the levels of Rantes were almost undetectable (close to zero). We suppose that the areas without regular hair growth have lost or already halt the chemotactic activity towards monocyte species.

Among these chemokines, two of them, IP-10 and MIP-1 $\alpha$, had significantly higher level in alopecia zone comparing to borderline area. Bearing this in mind we could speculate that higher IP-10 and MIP-1a level in alopecia vs. battle field zone may also regard as evidence of inflammatory process. In contrast, the lowest MIP-1 a level in occipital area and periumbilical adipose tissue could be a sign of silent follicular neighboring tissue metabolic activities. The measured protein concentration reflects completely distinct gene expression, and that inflammation governed by these two chemokines is still more pronounced in the alopecia zone than in the battle field zone. If we consider the occipital zone as a steady-state environment, and alopecia zone as a place of intensive inflammation, the borderline alopecia zone (actual battle field), should be a place with acute and active signaling molecules interplay judging by the levels of all examined protein (in the middle by values among three zones).

It is interesting that MCP-1 showed significant correlation only with eotaxin, and presents the only which were not in significant correlation with any other proteins analyzed in this current study. However, the variation in eotaxin level among scalp adipose tissue zones reflects continuous struggle in the borderline zone to preserve hair growth and to resist opposite forces, and it could be used for attraction and activation of different type of white blood cells.

Different inflammatory factors were mostly studied in the correlation with another type of alopecia, Alopecia Areata (AA). One of them, Tumor Necrosis Factor Alfa (TNF- $a$ ), has been found in sera and lesion biopsy samples from patients with AA. [19] The pathogenesis of AGA and AA is different, hence, as we expected, the levels of TNF- a were not significantly different in our samples. 
Since androgen imbalance and metabolism may be locally impaired by proinflammatory cytokines like EGF, transforming growth factor beta (TGF- $\beta$ ), Inteleukin - 1 (IL-1) and TNF-a, it could be possible that once the inflammatory process has been triggered, the androgenetic mechanism of alopecia could be locally amplified. [20] Therefore, it is not surprising that the levels of EGF in our AGA patient's group were significantly higher in scalp area than in periumbilical zone.

According to the laboratory and experimental studies, and contrary of the effect of EGF, growth factors such as HGF, VEGF, IGF, and PDGF increase the size of the hair follicle during hair development.

VEGF plays an important role in the system that restores the oxygen supply to tissue when the blood circulation is inadequate, e.g. hypoxic conditions. VEGF controls hair growth by angiogenesis in a mouse model. [21] In addition, VEGF has an important role in that process as it promotes perifollicular vascularization, hair growth rates, and increased follicle and hair size. On the other hand, the blockade of VEGF-mediated angiogenesis leads to impaired hair growth. [22] Our study showed that the VEGF level was significantly elevated in the alopecia area in comparison to other hair growing areas. Knowing that the effects of VEGF are dependent on a functional vascular system, and that its expression is upregulated, besides other skin conditions, in healing wounds and other skin diseases characterized by enhanced angiogenesis we hypothesize that these elevated levels in alopecia area are due to an insufficient perifollicular vascularization. [22] This finding may also indicate hypoxic background of the AGA which in turn activates inflammatory state in surrounding adipocytes and triggers "vicious cycle" in production of proinflammatory signals.

Probably the most investigated influence on hair growth has been of the PDGF. PDGF signaling is involved in the development of several organs and maintenance of adult tissues, including HF regeneration in the hair cycle. [23] Although the mechanism of how PDGF signaling promotes bulge cells activation is not clear, Festa et al. concluded that PDGF secreted by adipocyte precursor cells promoted hair growth. [24] It is interesting that Rezza et al [25] found that dermal PDGF signaling is not required for HF morphogenesis. Our results showed no difference in PDGF levels between the different zones of hair growth, but, additional analysis of larger patient group is necessary to confirm this.

The positive, strong correlation between all adipokines that have shown significant difference in alopecia area in comparison to others, could be a result of natural molecules' cognation, the mutual signals and factors responsible for its induction/generation or maybe the consequence of alopecia and its pathological influence on the specific metabolic paths and thus whole family of proteins.

ADSCs and their secretomes were proven to mediate diverse skin-regenerative effects, such as woundhealing, antioxidant protection, anti-wrinkling and whitening effects. $[26,27]$ With regard to paracrine actions, the limitations must not be overlooked. For instance, some cytokines or chemokines released from MSCs may be harmful, such as TNF-a and IL-6. This may be, besides rapid cell death upon transplantation, another explanation of the modest benefit of MSC transplantation observed in alopecia clinical trials. $[28,29]$ 
There is a far greater potential behind the regenerative treatments that has not been explored for AGA. The use of the ADSCs secretome as a therapeutic agent in AGA could be a promising new avenue. To avoid several of the limitations of cell-based approaches, ADSCs secretome in alopecia could be used as a personal approach to treat early changes (co-cultivation or/and co-transplantation of hair follicles and ADSCs secretome from the same person) or/and development patient-based secretome-dependant supplemental therapy as a paracrine effects of ADSCs on adipocyte-assisted HFs renewal. The application of adipose-derived stem cell constituent extract topical solution has been already proven to increase both hair density and thickness while maintaining adequate treatment safety. [30]

Won et al. studied the efficacy of ADSCs conditioned media (ADSC-CM) on hair growth and found out that ADSCs-derived proteins improve hair growth and protect human dermal papilla cells against cytotoxic injury caused by androgen and reactive oxygen species. Moreover, ADSC-CM induced the anagen phase and promoted hair growth in mice, and enhanced the elongation of hair shafts in ex vivo human hair organ cultures. [31] The growth factors released by ADSCs stimulate hair growth in ex vivo and in vivo animal models and may be feasible clinical therapeutic agents for the treatment of hair loss. [31]

However, growing evidence suggests that the secretion profile could be improved by preconditioning or genetic manipulation. As Liang et al. summarized, the paracrine action provides the opportunity to apply one trophic factor alone or in combination in cocktail therapy for disease-oriented treatment. [5] Thus, paracrine mechanisms of mesenchymal stem cell-based therapy hold great promise as a controllable, manageable, and feasible route, making the transition from bench to bedside possible. [32]

The limitation of our study is, besides the small sample size, the fact that, like the most of similar studies, it was devoted to the influence of separate growth factors that were detectable with the commercial kit that we used.

Up to our knowledge, this is the first study conducted in humans dealing with secretome of ADSC from different zones of scalp hair growth in patients with early stage of AGA. Knowledge of these signaling molecules and their pathways may be essential for achieving therapeutic goals and improving existing protocols for hair loss conditions as well as aiding in the search in the etiology of different types of hair follicle disorders. [33, 34]

\section{Conclusion}

Dermal white adipose tissue should be considered a target for any potential therapy that aims to modulate the hair growth, weather to promote or to remove unwanted hair.

Our study showed changes in the levels of EGF, VEGF, IL-6, MCP-1, MCP-3, IP-10, Eotaxin and MIP-1 $\alpha$ in different hair growing areas among patients with early stage of AGA, suggesting that these molecules and their signaling pathways play an essential role in the pathogenesis, and pointed out inflammatory background of AGA. 
Therefore, ADSC secretome has great potential as an alternative therapeutic strategy for hair regrowth in patients with androgenetic alopecia.

\section{Abbreviations}

ADSCs - Adipose Derived Stem Cells; HF - Hair Follicle; dWAT - dermal White Adipose Tissue; AGA Androgenetic Alopecia; MSCs - Mesenchymal stem cells; DMEM F12 - Dulbecco's Modified Eagle Medium; DPBS - Dulbecco's Phosphate-Buffered Saline; ADSC-CM - Adipose Derived Stem Cells conditioned media; FBS - Fetal Bovine Serum; ROC - Receiver Operating Characteristics; DP - Dermal Papilla; FUE - Follicular Unit Extraction; IL-6 - Interleukin-6; SVF - Stromal Vascular Fraction; EGF Endothelial Growth Factor; VEGF - Vascular Endothelial Growth Factor; MCP-1- Monocyte Chemoattractant Protein-1; MCP-3 - Monocyte Chemotactic Protein-3; IP-10 - Interferon gamma-inducible Protein; MIP-1a - Macrophage Inflammatory Protein - 1 alpha; HGF - Hepatocyte Growth Factor; IGF Insulin-like Growth Factor; PDGF - Platelet Derived Growth Factor; TNF-a - Tumor Necrosis Factor Alfa; AA - Alopecia Areata; TGF- $\beta$ - Transforming Growth Factor Beta; IL-1 - Inteleukin-1

\section{Declarations}

\section{Ethics approval and consent to participate}

This study was approved by the Ethics Committee of the Hospital Colic, Belgrade. The procedure was conducted in accordance with the Declaration of Helsinki. All participants provided written informed consent prior to inclusion in the study.

\section{Consent for publication}

All patients signed the consent form.

\section{Availability of data and materials}

Not applicable.

\section{Competing interests}

The authors declare that they have no competing interests

\section{Funding}

No funding has been received.

\section{Authors' contributions}

KA contributed to the experimental conceptualization and design, performed all biopsies and was a major contributor in writing the manuscript. Both KA and AK analyzed and interpreted the patient data. AK 
provided considerable manuscript review. IE performed the histological examination of the biopsies and organized all data. All authors read and approved the final manuscript.

\section{Acknowledgements}

Not applicable.

\section{References}

1. Schmidt B, Horsley V. Unravelling hair follicle-adipocyte communication. Exp Dermatol. 2012;21(11):827-30. doi: 10.1111/exd.12001.

2. Kruglikov IL, Zhang Z, Scherer PE. The Role of Immature and Mature Adipocytes in Hair Cycling. Trends Endocrinol Metab. 2019;30(2):93-105. doi: 10.1016/j.tem.2018.11.004. Epub 2018 Dec 14.

3. Baldari S, Di Rocco G, Piccoli M, et al. Challenges and Strategies for Improving the Regenerative Effects of Mesenchymal Stromal Cell-Based Therapies. Int J Mol Sci. 2017;18(10). pii: E2087. doi: 10.3390/ijms18102087.

4. Doorn J(1), Moll G, Le Blanc K, van Blitterswijk C, de Boer J. Therapeutic applications of mesenchymal stromal cells: paracrine effects and potential improvements. Tissue Eng Part B Rev. 2012;18(2):101-15. doi: 10.1089/ten.TEB.2011.0488. Epub 2011 Dec 22.

5. Liang X, Ding Y, Zhang Y, Tse HF, Lian Q. Paracrine mechanisms of mesenchymal stem cell-based therapy: current status and perspectives. Cell Transplant. 2014;23(9):1045-59. doi: $10.3727 / 096368913 \times 667709$.

6. Phinney DG(1), Pittenger MF(2). Concise Review: MSC-Derived Exosomes for Cell-Free Therapy. Stem Cells. 2017 Apr;35(4):851-858. doi: 10.1002/stem.2575. Epub 2017 Mar 10.

7. Shin $\mathrm{H}(1)$, Won $\mathrm{CH}(2)$, Chung WK(3), Park BS(3). Up-to-date Clinical Trials of Hair Regeneration Using Conditioned Media of Adipose-Derived Stem Cells in Male and Female Pattern Hair Loss. Curr Stem Cell Res Ther. 2017;12(7):524-530. doi: 10.2174/1574888X12666170504120244.

8. Shin $H(1)$, Ryu HH(2), Kwon O(2), Park BS(3), Jo SJ(1). Clinical use of conditioned media of adipose tissue-derived stem cells in female pattern hair loss: a retrospective case series study. Int J Dermatol. 2015;54(6):730-5. doi: 10.1111/ijd.12650. Epub 2015 Mar 16.

9. Mahé YF(1), Michelet JF, Billoni N, Jarrousse F, Buan B, Commo S, Saint-Léger D, Bernard BA. Androgenetic alopecia and microinflammation. Int J Dermatol. 2000;39(8):576-84.

10. Gheisari $M(1)$, Hamidi $A B(1)$, Hamedani $B(1)$, Zerehpoush FB(2). Androgenetic alopecia; An attempt to target microinflammation. Dermatol Ther. 2020;33(2):e13266. doi: 10.1111/dth.13266. Epub 2020 Feb 24.

11. Ramos PM(1), Brianezi G(2), Martins AC(2), da Silva MG(2), Marques ME(2), Miot HA(1). Apoptosis in follicles of individuals with female pattern hair loss is associated with perifollicular microinflammation. . Int J Cosmet Sci. 2016;38(6):651-654. doi: 10.1111/ics.12341. Epub 2016 Jun 1. 
12. Sadgrove $\mathrm{NJ}(1)$. The new paradigm for androgenetic alopecia and plant-based folk remedies: $5 a-$ reductase inhibition, reversal of secondary microinflammation and improving insulin resistance. $J$ Ethnopharmacol. 2018;227:206-236. doi: 10.1016/j.jep.2018.09.009. Epub 2018 Sep 6.

13. Jaworsky C(1), Kligman AM, Murphy GF. Characterization of inflammatory infiltrates in male pattern alopecia: implications for pathogenesis. Br J Dermatol. 1992 Sep;127(3):239-46.

14. Young JW(1), Conte ET, Leavitt ML, Nafz MA, Schroeter AL. Cutaneous immunopathology of androgenetic alopecia. J Am Osteopath Assoc. 1991;91(8):765-71.

15. Ben-Baruch A, Xu L, Young PR, Bengali K, Oppenheim JJ, Wang JM. Monocyte chemotactic protein-3 (MCP3) interacts with multiple leukocyte receptors. C-C CKR1, a receptor for macrophage inflammatory protein-1 alpha/Rantes, is also a functional receptor for MCP3. J Biol Chem. 1995;270(38):22123-22128. doi:10.1074/jbc.270.38.22123

16. Kabashima H, Yoneda M, Nagata K, Hirofuji T, Maeda K. The presence of chemokine (MCP-1, MIP1alpha, MIP-1beta, IP-10, RANTES)-positive cells and chemokine receptor (CCR5, CXCR3)-positive cells in inflamed human gingival tissues. Cytokine. 2002;20(2):70-77. doi:10.1006/cyto.2002.1985

17. Boström EA, Kindstedt E, Sulniute R, et al. Increased Eotaxin and MCP-1 Levels in Serum from Individuals with Periodontitis and in Human Gingival Fibroblasts Exposed to Pro-Inflammatory Cytokines. PLoS One. 2015;10(8):e0134608. Published 2015 Aug 4. doi:10.1371/journal.pone.0134608

18. Yadav A, Saini V, Arora S. MCP-1: chemoattractant with a role beyond immunity: a review. Clin Chim Acta. 2010;411: 1570-1579. 10.1016/j.cca.2010.07.006

19. Kasumagic-Halilovic E, Prohic A, Cavaljuga S. Tumor necrosis factor-alpha in patients with alopecia areata. Indian J Dermatol. 2011;56(5):494-6. doi: 10.4103/0019-5154.87124.

20. Kasasa SC(1), Soory M. The effect of interleukin-1 (IL-1) on androgen metabolism in human gingival tissue (HGT) and periodontal ligament (PDL). J Clin Periodontol. 1996;23(5):419-24.

21. Yano K(1), Brown LF, Detmar M. Control of hair growth and follicle size by VEGF-mediated angiogenesis. J Clin Invest. 2001;107(4):409-17.

22. Yano K, Brown LF, Detmar M. Control of hair growth and follicle size by VEGF-mediated angiogenesis. J Clin Invest. 2001;107(4):409-17.

23. Peus D, Peus D, Pittelkow MR. Growth factors in hair organ development and the hair growth cycle. Dermatol Clin. 1996;14(4):559-572. doi:10.1016/s0733-8635(05)70384-3

24. Festa E, Fretz J, Berry R, et al. Adipocyte lineage cells contribute to the skin stem cell niche to drive hair cycling. Cell. 2011;146:761-71.

25. Rezza A, Sennett R, Tanguy M, Clavel C, Rendl M. PDGF signalling in the dermis and in dermal condensates is dispensable for hair follicle induction and formation. Exp Dermatol. 2015;24(6):46870. doi: 10.1111/exd.12672. Epub 2015 Mar 25.

26. Park BS, Kim WS, Choi JS, et al. Hair growth stimulated by conditioned medium of adipose-derived stem cells is enhanced by hypoxia: evidence of increased growth factor secretion. Biomed Res. 2010;31(1):27-34. 
27. Kim WS(1), Park BS, Sung JH. Protective role of adipose-derived stem cells and their soluble factors in photoaging. Arch Dermatol Res. 2009; 301(5):329-36. doi: 10.1007/s00403-009-0951-9. Epub 2009 Apr 26.

28. Kuka G, Epstein J, Aronowitz J, et al. Cell Enriched Autologous Fat Grafts to Follicular Niche Improves Hair Regrowth in Early Androgenetic Alopecia. Aesthet Surg J. 2020 Feb 5. pii: sjaa037. doi: 10.1093/asj/sjaa037. [Epub ahead of print]

29. Perez-Meza D, Ziering C, Sforza M, et al. Hair follicle growth by stromal vascular fraction-enhanced adipose transplantation in baldness. Stem Cells Cloning. 2017;10:1-10. doi: 10.2147/SCCAA.S131431. eCollection 2017.

30. Tak YJ, Lee SY, Cho AR, Kim YS. A randomized, double-blind, vehicle-controlled clinical study of hair regeneration using adipose-derived stem cell constituent extract in androgenetic alopecia. Stem Cells Transl Med. 2020;10.1002/sctm.19-0410. doi:10.1002/sctm.19-0410

31. Won $\mathrm{CH}(1)$, Park GH(2), Wu X(3), et al. The Basic Mechanism of Hair Growth Stimulation by Adiposederived Stem Cells and Their Secretory Factors. Curr Stem Cell Res Ther. 2017;12(7):535-543. doi: 10.2174/1574888X12666170829161058.

32. Liang X, Ding Y, Zhang Y, Tse HF, Lian Q. Paracrine mechanisms of mesenchymal stem cell-based therapy: current status and perspectives. Cell Transplant. 2014;23(9):1045-1059. doi:10.3727/096368913X667709

33. Millar SE. Molecular mechanisms regulating hair follicle development. J Invest Dermatol. 2002;118(2):216-25.

34. Rishikaysh P, Dev K, Diaz D, et al. Signaling involved in hair follicle morphogenesis and development. Int J Mol Sci. 2014 Jan 22;15(1):1647-70. doi: 10.3390/ijms15011647.

\section{Tables}

Table 1. Sociodemographic, antropometric and clinical patients' data

\begin{tabular}{|lll|}
\hline Variable & Mean \pm SD $/ \mathrm{No}(\%)$ & Range (min-max) \\
\hline Age (years) & $40.3 \pm 10.5$ & $33-57$ \\
\hline BMI $\left(\mathrm{kg} / \mathrm{m}^{2}\right)$ & $24.8 \pm 5.0$ & $20-34$ \\
\hline Smoking (yes/no) & $1 / 5(16.7 \%)$ & $/$ \\
\hline Therapy & $? ? ?$ & $/$ \\
\hline Comorbidity & $? ? ?$ & $/$ \\
\hline
\end{tabular}

SD- standard deviation, No-number

Table 2. Correlation between different proteins (cytokines, adipokines, signaling molecules...) 


\begin{tabular}{|c|c|c|c|c|c|c|c|}
\hline Parameter & $\begin{array}{c}\text { EOTAXI } \\
\mathrm{N}\end{array}$ & MCP-3 & IL-6 & IP-10 & MCP-1 & MIP-1A & VEGF \\
\hline EGF & $0,724 * * *$ & $\begin{array}{r}0,869 * * \\
*\end{array}$ & $\begin{array}{r}0,832^{* * *} \\
*\end{array}$ & $0,534 * *$ & 0,321 & $0,574 * *$ & $0,529 * *$ \\
\hline $\begin{array}{l}\text { EOTAXI } \\
\mathrm{N}\end{array}$ & / & $\begin{array}{r}0,736 * * \\
* \\
\end{array}$ & $0,645^{* *}$ & $0,641^{* *} *$ & $0,421 *$ & $\begin{array}{r}0,681^{* *} \\
*\end{array}$ & $0,524 * *$ \\
\hline MCP-3 & $/$ & $/$ & $0,844^{* *}$ & $0,577 * *$ & 0,311 & $0,620 * *$ & $\begin{array}{r}0,700 * * \\
*\end{array}$ \\
\hline IL-6 & $/$ & 7 & 7 & $0,608^{* *}$ & 0,400 & $0,452 *$ & $\begin{array}{r}0,689 * * \\
*\end{array}$ \\
\hline IP-10 & $/$ & / & $/$ & $/$ & 0,346 & $\begin{array}{r}0,669 * * \\
*\end{array}$ & $0,639 * *$ \\
\hline MCP-1 & $/$ & / & $/$ & $/$ & 1 & 0,273 & 0,387 \\
\hline MIP-1A & 7 & 7 & $/$ & $/$ & 7 & / & $\begin{array}{r}0,687^{* *} \\
*\end{array}$ \\
\hline
\end{tabular}

Table 3. Predicted probability from VEGF and MCP-1 combined in ROC analysis testing diagnostic accuracy for alopecia positivity status (reference group was occipital zone adipose tissue)

\begin{tabular}{|lll|}
\hline AUC (SE) & $95 \% \mathrm{Cl}$ & $\mathrm{P}$ \\
\hline $0,889(0,117)$ & $0,580-0,994$ & 0,0008 \\
\hline
\end{tabular}

Figures 


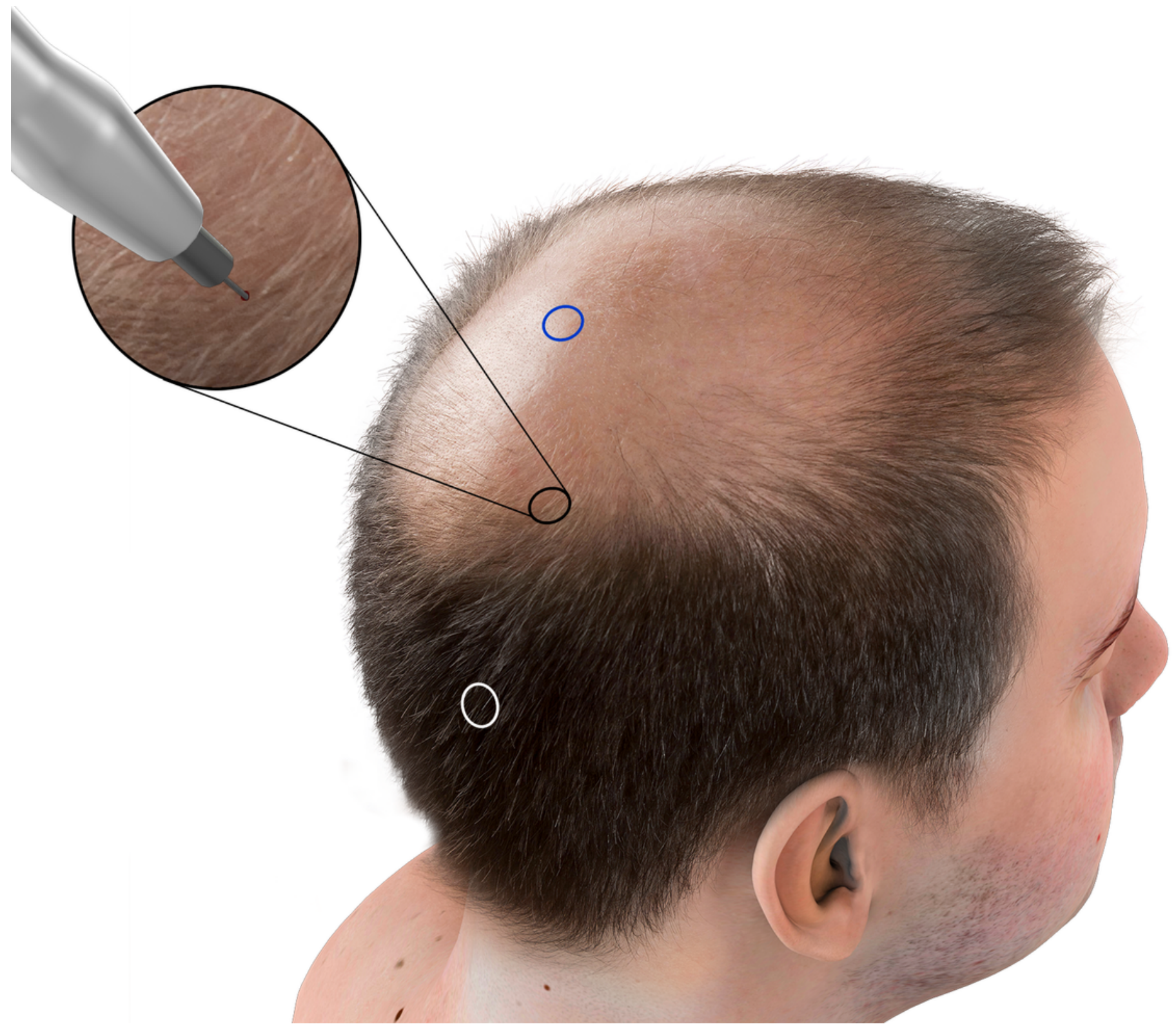

\section{Figure 1}

Scalp location of performed 1-mm punch biopsies: 1. White circle- Occipital zone (donor area for hair transplants), normal hair growth area; 2. Black circle - Border line zone ("battle field area"), area where there is still a hair growth, but the hair is thinner and scarce; 3 . Blue circle - Alopecia zone ("Iost battle field"), area with no hair growth, recipient area for hair transplants 

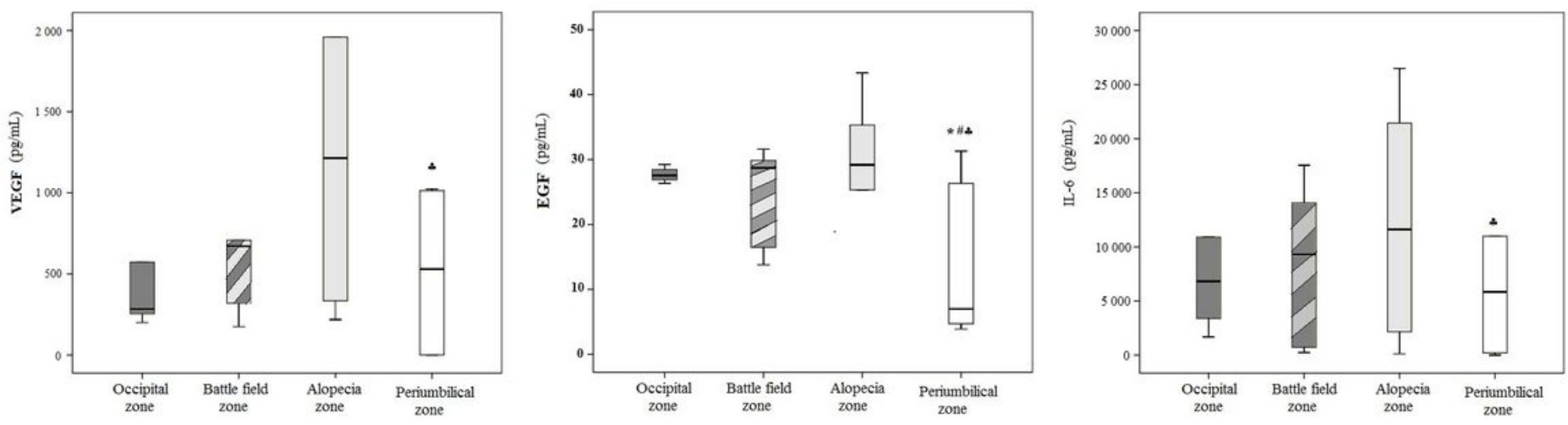

Figure 2

Stimulators of cell growth and differentiation (EGF and VEGF) and proinflammatory marker (IL-6) according to different alopecia zones and in periumbilical zone (Wilcoxon's paired test, symbols: *, \#, $p<0.05$ vs. alopecia zone, battle field zone, occipital zone, respectively)
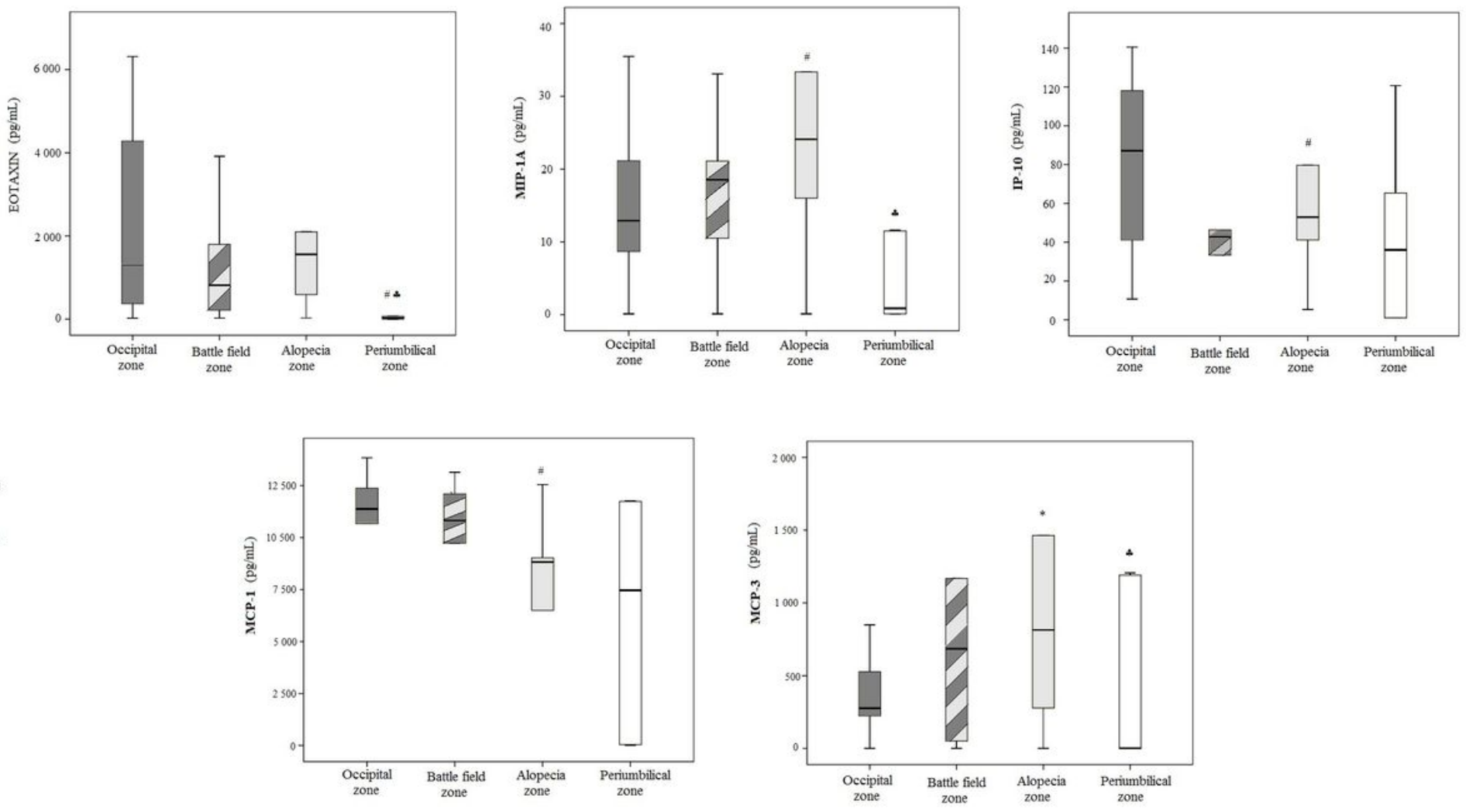

Figure 3

Chemokines (Eotaxin, MIP-1A, IP-10, MCP-1, MCP-3) according to different alopecia zones and in periumbilical zone (Wilcoxon's paired test, symbols: * \#, \& $p<0.05$ vs. alopecia zone, battle field zone, occipital zone, respectively) 